\title{
Extra gonadal sclerosing stromal tumour in the transverse mesocolon
}

\author{
Samuel Mensah ${ }^{1}$, Ishmael Kyei ${ }^{1}$, Michael Ohene - Yeboah $^{2}$ and Ernest Adjei ${ }^{3}$ \\ ${ }^{1}$ Directorate of Surgery, Komfo Anokye Teaching Hospital, P O Box 1934, Kumasi, Ghana ${ }^{2}$ Department of \\ Surgery, University of Ghana School of Medicine and Dentistry, Accra, Ghana ${ }^{3}$ Department of Pathology, \\ Komfo Anokye Teaching Hospital, Kumasi, Ghana
}

DOI: $h$ ttp://dx.doi.org/10.4314/gmj.v50i1.9

Corresponding author: Dr. Samuel Mensah $\quad$ E-mail: skwabenamensah@yahoo.com

Conflict of interest: None declared

\section{SUMMARY}

Sclerosing stromal tumour (SST) is a rare benign sex cord stromal tumour of the ovary. We report a case of sclerosing stromal tumour of the mesentery in a 32-year-old Para one who presented with intra abdominal mass, menstrual irregularity and secondary infertility. Histopathology and immunohistochemistry of the completely excised tumour was consistent with sclerosing stromal tumour, immunoreactive only to vimentin. No ovarian tissue was found in the sectioned tumour. Her menses became regular and she conceived 3 months after complete excision and delivered after 9 months. Hormonal assay was not done because SST was least suspected. From literature this is the first case of SST in the transverse mesocolon reported in the West African subregion, and may probably be one of the rare cases of hormonally active SST.

Keywords: Extragonadal, sclerosing stromal tumour, Hormonally active, secondary infertility, Ghana

\section{INTRODUCTION}

Sclerosing stromal tumours (SST) are rare benign ovarian neoplasms. They are a subtype of ovarian sex cord stromal tumour which usually occurs in the second and third decades of life. ${ }^{1,2}$ Extra gonadal sex cord tumours are extremely rare. ${ }^{3}$ These are mostly granulosa cell tumours which are thought to arise from extra ovarian mesenchyme of the genital ridge. ${ }^{2}$

\section{CASE REPORT}

We present a case of primary mesenteric extra gonadal sclerosing stromal tumour. A thirty-two year old Para 1, presented with epigastric mass of 4 years duration associated with occasional dull pain. The mass was first noticed 8 weeks into pregnancy. She had spontaneous abortion at 12 weeks gestation. She complained of irregular menses and did not become pregnant again after the abortion.

Examination revealed a well - looking young woman. On examination of the abdomen, there was a bulge in the epigastrium. There was an intra abdominal mass palpated in the epigastric region of the abdomen that measured approximately $10 \times 7 \mathrm{~cm}$. The mass was firm, non-tender, mobile and non-pulsatile.
CT scan revealed a heterogeneously enhancing mass lying between the liver and the right kidney measuring $8.2 \times 9.1 \times 9.4 \mathrm{~cm}$ in close contact with the liver but not within it. A diagnosis of retroperitoneal leiomyosarcoma was made with normal appearance of all other viscus. There were no abnormalities of the ovaries.

Laparotomy revealed a solid tumour in the transverse mesocolon, abutting the duodenum (Figure 1). Complete excision was done. She had an uneventful post operative period and conceived 3 months after surgery and delivered 9 months later.

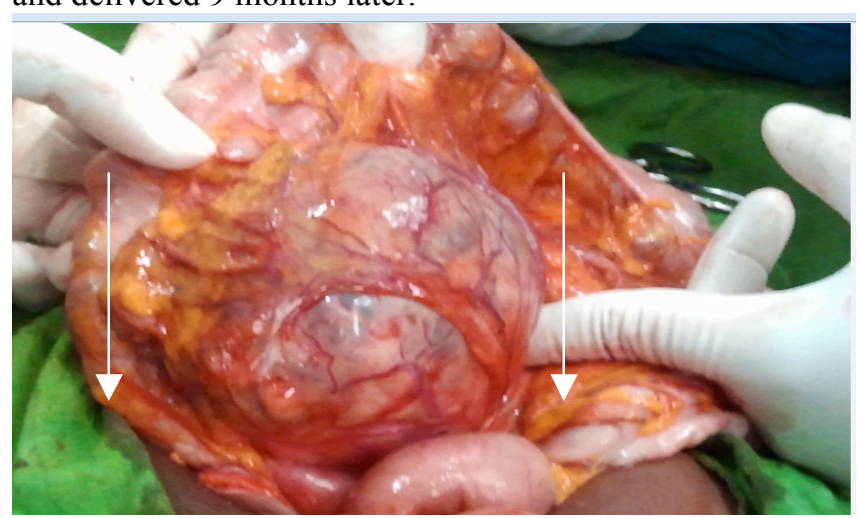

Figure 1 Mesenteric tumour at laparotomy 


\section{Case Report}

Gross examination showed a $10 \times 8 \times 6 \mathrm{~cm}$ soft to firm brownish encapsulated abdominal tissue. On gross pathological examination, cut surfaces showed a heterogeneous appearance with brownish and yellowish areas and haemorrhages within the tumour.

Microscopy showed a well circumscribed tumour with lobular pattern. There was fibrosis and marked vascularity. The tumour also showed hypo and hyper cellular areas with prominent vessels (Figure 2). There was dual
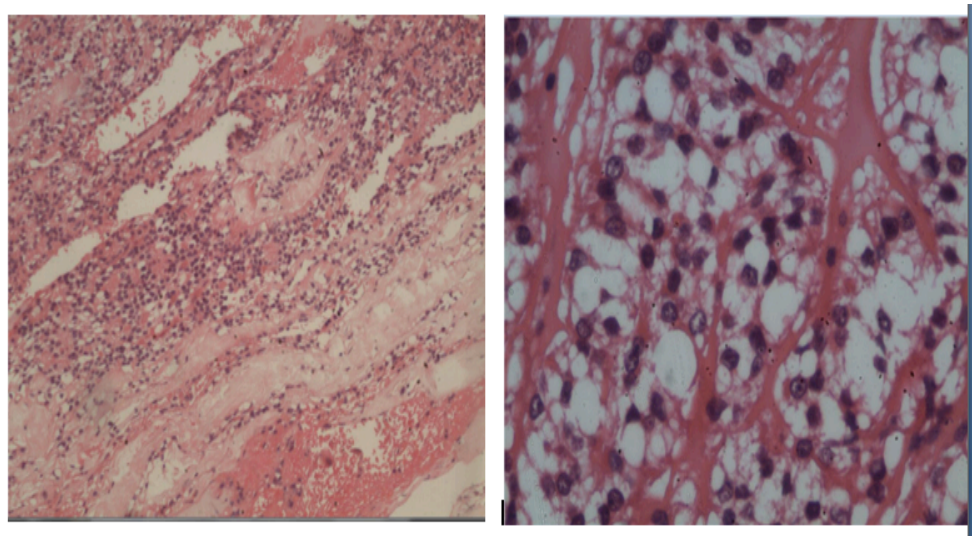

tumour cell population.

Figure 2 Sclerosing stromal tumour (H\&E) showing a heterogeneous picture with hypo and hyper vascular regions on the left and Lipid laden cells to the right.

One type was spindle cells while the other population was round to oval cells which were pale and had some signet ring- like cell appearance. No mitotic figures were identified. A diagnosis of a sclerosing stromal tumour of ovary was made. There was no ovarian tissue in and around sections of the tumour examined. Immunohistochemical study of the resected mass showed immunoreactivity for only Vimentin (Figure 3).

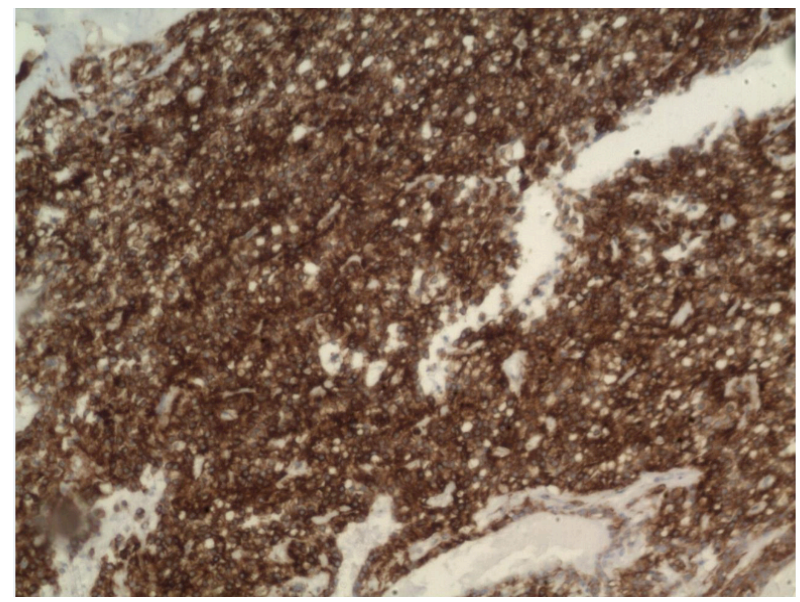

Figure 3 Tumour cells immunoreactive to vimentin
Other markers such as CD 45(lymphocyte common Antigen), AE1/AE2 (pan cytokeratin), CD 117, Calretinin, AFP, Inhibin, S 100, SMA, myogenin were negative. CA 125 assay was within normal limits - 23.1U/ml (0.0-35.0)

\section{DISCUSSION}

Sclerosing stromal tumour (SST) is a benign type of ovarian sex cord stromal tumours. It constitutes about $5 \%$ of sex cord stromal tumours of the ovary. ${ }^{4}$ It occurs in a younger age group compared with other types of stromal tumours, usually the $2^{\text {nd }}$ and $3^{\text {rd }}$ decades. ${ }^{2,4,5}$ This rare tumour was first described as a distinct entity in 1973 by Chalvardjan and Sully. ${ }^{6}$ Gross examination of this type of tumour shows solid or solid and cystic gray to white cut surface with occasional yellow areas. ${ }^{2,7}$

Microscopic picture of SST is heterogeneous unlike other stromal tumours which show homogenous histological picture ${ }^{2}$. Histological features include cellular pseudo lobules, prominent interlobular fibrosis, marked vascularity and dual cell population, collagen producing spindles cells and lipid containing round and ovoid cells. $^{2,4,5}$

Several immunohistochemical markers are used to diagnose SST. These include Inhibin, Calretinin, CD 99, Vimentin, desmin and smooth muscle (s-m) actin. They however have variable expressions. ${ }^{2,9}$ SST are usually vimentin positive, s-m actin positive, inhibin positive or negative, calretinin positive or negative, desmin positive or negative, and pancytokeratin negative. ${ }^{9}$ In this case only vimentin was positive.

SST has varied clinical presentations. The commonest symptom is menstrual irregularity which is reported to disappear after surgical excision of the tumour. ${ }^{2,8,9}$ Other symptoms include pelvic pain, and non specific pain related to ovarian mass. The tumours may, in rare cases, cause masculinisation, anovulation and infertility. ${ }^{2,10,11}$

It is extremely rare for primary sex cord stromal tumours to occur at extra ovarian sites. The commonest extra ovarian primary sex cord tumours reported in literature is granulosa cell tumour. ${ }^{12}$ In 2008 Trabelsi et al reported the first case of primary mesenteric sertoli Leydig cell tumour. ${ }^{12}$ Naniwadekar et al in 2010 also reported an extra ovarian granulosa cell tumour in mesentery. ${ }^{13}$ In 2013, Moktari et al published the first case of sclerosing stromal tumour in an extra ovarian location; between the bladder and the uterus. ${ }^{2}$ 
This appears to be the only case of extraovarian sclerosing stromal tumour reported, as the one reported by Andrade et al in 2001 was found in an accessory ovary but attached to the ovary. ${ }^{14}$

Here we report an extra ovarian SST located in the transverse mesocolon, the first in this location and in the West African sub region. Gross examination showed a heterogeneous mass. This heterogeneity can differentiate SST from other stromal tumours.

In spite of the benign nature of SST, few are reported to be hormonally active and present with menstrual irregularity and infertility. ${ }^{2,8-10}$ In this case the patient presented with irregular menses and secondary infertility. Complete excision of the tumour resulted in normalisation of the menses and subsequent conception and delivery. Similar results have been reported with the disappearance of menstrual irregularity with excision of the tumour. ${ }^{2,8,9}$ This could be a rare case of hormonally active SST. Hormonal assay was not done because SST was least suspected in the mesentery. The uneventful post excision period of 2 years confirms that complete excision is enough for cure without strict follow up and adjuvant treatment.

\section{REFERENCES}

1. Haroon S, Zia A, Idrees R, Memon A, Fatima S, Kayani N. Clinicopathological spectrum of ovarian sex cord-stromal tumors; 20 years' retrospective study in a developing country. J Ovarian Res. 2013;6(1):87.

2. Mokhtari M, Akbarzadeh-Jahromi M, Sari-Aslani F, Hamedi B, Bagheri M, Torfehnezhad P. Extragonadal sclerosing stromal tumor: A rare case report. J Obstet Gynaecol Res. 2014 Mar 1;40(3):883-6.

3. Keitoku M, Konishi I, Nanbu K, Yamamoto S, Mandai M, Kataoka N, et al. Extraovarian sex cordstromal tumor: case report and review of the litera- ture. Int J Gynecol Pathol Off J Int Soc Gynecol Pathol. 1997 Apr;16(2):180-5.

4. Khanna M, Khanna A, Manjari M. Sclerosing Stromal Tumor of Ovary: A Case Report. Case Rep Pathol. 2012 Oct 12;2012:e592836.

5. Ghada Elsayed Esheba, Teri A Longacre. Sex Cord Stromal Ovary Tumor Pathology. 2013 Sep 26 [cited 2014 May 8]; Available from: http://emedicine.medscape.com/article/1627984overview

6. Chalvardjian A, Scully RE. Sclerosing stromal tumors of the ovary. Cancer. 1973;31(3):664-70.

7. Kaygusuz EI, Cesur S, Cetiner H, Yavuz H, Koc N. Sclerosing stromal tumour in young women: clinicopathologic and immunohistochemical spectrum. $J$ Clin Diagn Res JCDR. 2013 Sep;7(9):1932-5.

8. Fotedar V, Gupta MK, Seam RK. Sclerosing stromal tumour of the ovary: case study. South Afr J Gynaecol Oncol. 2012;4(2):66-8.

9. Peng H-H, Chang T-C, Hsueh S. Sclerosing stromal tumor of ovary. Chang Gung Med J. 2003 Jun;26(6):444-8.

10. Akbulut M, Colakoglu N, Soysal ME, Duzcan SE. Sclerosing stromal tumor of the ovary: Report of a case and review of the literature. Agean Pathol J. 2004;1:84-9.

11. Mikami M, Fukuchi T, Takehara K, Komiyama S, Suzuki A, Hirose T. Tumor imprint cytology of sclerosing stromal tumor of the ovary. Diagn Cytopathol. 2003;28(1):54-7.

12. Trabelsi A, Ben Abdelkarim S, Hadfi M, Fatnaci R, Stita W, Sriha B, et al. Primary mesenteric sertolileydig cell tumor: a case report and review of the literature. J Oncol. 2008;2008:619637.

13. Naniwadekar MR, Patil NJ. Extraovarian Granulosa Cell Tumor of Mesentery: A Case Report. Pathol Res Int. 2010 Mar 4;2010:e292606.

14. Andrade LA, Gentilli AL, Polli G. Sclerosing stromal tumor in an accessory ovary. Gynecol Oncol. 2001 May;81(2):318-9. 